\title{
Three-Mode Principal Components Analysis of Semantic Differential Data: The Case of a Triple Personality
}

\author{
Pleter M. Mroonenberg \\ University of Leiden, The Netherlands
}

This paper shows how three-mode principal components analysis can be useful for the analysis of semantic differential ratings, in particular because no summation is necessary over any one mode. The use of "joint plots" (a variant of the biplot) and sums-ofsquares interpretations is explained and illustrated.

The aim of this paper is to show the power of three-mode principal components analysis in constructing one unified description of data collected under different circumstances, but referring to the same underlying structure. This is illustrated with data from probably the most famous case of a multiple personality: Eve White, Eve Black, and Jane (Thigpen \& Cleckley, 1954). Osgood and Luria (1954/1969) published scores on semantic differential scales for each personality of this case at two occasions (Testings $\mathbb{I}$ and $I$ ). In essence the data set has four modes, that is, personalities, testings, concepts, and scales. This paper, however, treats the data as three-mode data, and the 6 administrations of 10 scales by 15 concepts are the data on which this reanalysis is based. The example is used to show how individual differences in the use of semantic differential scales and concept-scale in-

APPLIED PSYCHOLOGICAL MEASUREMENT

Vol. 9, No. 1, March 1985, pp. 83-94

(C) Copyright 1985 Applied Psychological Measurement Inc. 0146-6216/85/010083-12\$1.85 teractions can be analyzed with three-mode principal components analysis.

The histories of three-mode principal components (or factor) analysis (or three-mode analysis, for short) and the semantic differential technique (Heise, 1969; Osgood, Suci, \& Tannenbaum, 1957; Snider \& Osgood, 1969) have been relatively closely linked since the introduction of the former by Tucker (1963). Both procedures were developed mainly at the University of Illinois, and the first more or less elementary exposition of three-mode analysis used the semantic differential as an illustration (Levin, 1965). Tucker, Osgood, Triandis, and others have published at least twenty applications of three-mode analysis to semantic and behavioral differential data (see Kroonenberg, 1983a, for annotated references).

A central focus in such studies is the dimensionality of the subject space, especially to determine whether averaging over subjects is appropriate. Most studies report more than one subject component, thus indicating the existence of individual differences in the use of concept, scales, and their interactions. The example to be discussed is especially interesting and fairly unique in the sense that the semantic differentials have been produced by a single person with three personalities. As will become apparent, the interpersonality differences are substantial, but the use of three-mode analysis allows the differences to be described by 
one single set of components for concepts or concept space, and one single scale space. The differences between the personalities will be seen to lie in the different ways in which they combine the common scale and concept spaces.

Whereas virtually all previous studies combining three-mode analysis and semantic differential data used Tucker's (1966) methods of analysis, the analysis presented here uses the least squares estimation procedures as developed by Kroonenberg and de Leeuv (1980; see also Kroonenberg, 1983b), and implemented in the TUCKALS3 program developed by Kroonenberg (1981). This new method has the advantage of allowing more detailed interpretation of the core matrix and of the fit of the model. Some new interpretational aids are used which have not been previously applied in threemode analysis of semantic differential data, the first of which allows simultaneous portrayal of concepts and scales, concepts and subjects, or subjects and scales, and the second the assessment of relative fit of any entity in the data set.

First, a short methodological appraisal is given of Osgood and Luria's analyses. The criticisms of their analyses aftempt to show how newer methodologies can be used to evaluate and extend their approach to the problem.

The form of semantic differential used in the study of the triple personality of Eve White, Eve Black, and Jane consists of the scales:

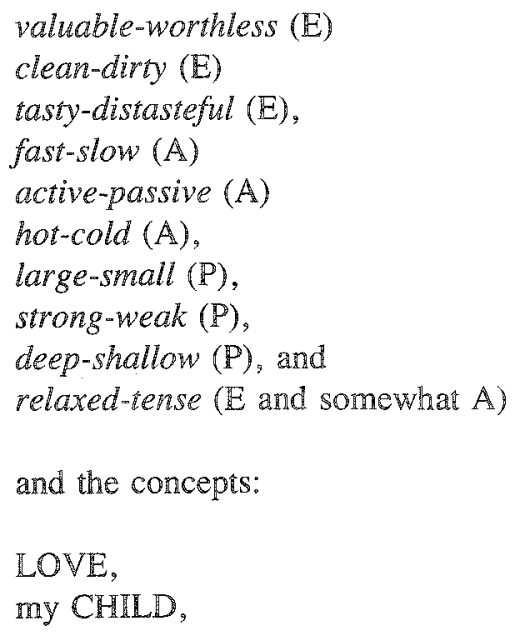

my DOCTOR,

$\mathbb{M E}$,

my JOB,

mental SICKNESS,

PEACE of mind,

FRAUD,

mY SPOUSE,

SElf-CONTROL, HATRED, mY $\mathbb{F A T H E R}$, MY MOTHER, CONFUSION, and SEX.

The Evaluation $(\mathbb{E})$, Potency (P), and Activity (A) indications for the scales are taken from Osgood and Luria (1954/1969, p. 506).

In order to represent the semantic structure of an individual in graphic form, Osgood and Luria performed the following steps for each administration: (1) computed a factor analysis on the scales to investigate a possible EPA structure; (2) computed generalized distances between concepts of "differences in meaning" from the factor scores for each pair of concepts, yielding a symmetric matrix with distances; and (3) plotted the principal components of this distance matrix. In light of present methodological advances this procedure is already rather indirect for each administration separately, but it is certainly so when a numerical comparison is desired of the administrations either for the concepts or for the scales, or for both.

In a later discussion of the Osgood and Luria (1954/1969) paper, Osgood et al. (1957) presented the three (rotated) factors for the scale spaces of the first testings (I) of Eve White, Eve Black, and Jane. These factor loadings showed a strong first rotated factor $(49 \%, 59 \%$, and $48 \%$ explained variation for the personalities, respectively) on which mearly all scales load positively, and which was interpreted as a "general" evaluative factor. The second and third factors resembled each other far less (as shown by their Spearman correlations of $.56, .14$, and .59 for the second factors, and .87 , .24 , and .21 for the third factors, respectively), but Osgood et al. saw sufficient similarities in them to 
state, "we have evidence, then, for essentially the same three major factors operating in the several personalities of this disturbed patient, although there is considerable shifting in meanings of specific scales between personalities . .." (p. 262). Inspection of their factor loadings and the correlations between them, however, shows that they present insufficient evidence for their assertions. In addition, it is questionable how useful the statement about shifting scales is without reference to the concepts to which the dimensions and scales apply. Because of this, it should be interesting to look at the data in their entirety, and to investigate the differences and similarities between the personalities.

\section{There-Node Hoincipal Components Analysis}

The primary purpose of three-mode principal components analysis in semantic differential studies is to study differences between individuals in their use of scales and concepts. Previous factor analyses have often been performed on the subjects-by-scales matrix summed over concepts, or on the subjects-by-concepts matrix summed over scales. Each of these types of analysis constitutes a reduction of the $(I \times J \times K)$ three-way or threemode data matrix, $\mathbb{Z}=\left(z_{i j k}\right)$, of concepts by scales by subjects, to a two-way matrix by summing over one mode. Thereby, it is implicitly assumed that the eliminated mode consists of mere replications without interactions with the other two modes.

In three-mode principal components analysis separate component loadings are simultaneously determined for subjects, concepts, and scales. In the technique there is no simple equivalent for the component (or factor) scores; in contrast, the model on which the technique is based has a set of parameters which has no straightforward analogue in standard principal components analysis, namely the core matrix. As its name suggests, the core matrix is the most fundamental part of the model, and it contains the information about the relationships between the components of the concepts, scales, and subjects. More formally the model may be expressed as

$\mathbb{Z}=\mathbb{A}\left(\mathbb{B}^{\prime} \otimes \mathbb{C}^{\prime}\right)+\mathbb{E}$,

$$
\text { where } \begin{aligned}
= & \left(a_{i p}\right) \text { is the }(I \times s) \text { matrix of } \\
& \text { concept loadings, } \\
= & \left(b_{j q}\right) \text { is the }(J \times \mathbb{T}) \text { matrix of scall } \\
& \text { loadings, } \\
\mathbb{C}= & \left(c_{k r}\right) \text { is the }(K \times u) \text { matrix of } \\
& \text { subject loadings, } \\
\mathbb{G}= & \left(g_{p q r}\right) \text { is the }(s \times t \times u) \text { core } \\
& \text { matrix, and } \\
\mathbb{E}= & \left(e_{i j k}\right) \text { is the }(\mathbb{I} \times J \times K) \text { three- } \\
& \text { mode matrix of residuals. }
\end{aligned}
$$

The expression $B(\mathbb{C}$ refers to the right Kronecker product of matrices, $\mathbb{B} \otimes C=\left(b_{j q} \mathbb{C}\right)$. Another way of expressing the model is to say that a score $z_{i j k}$ for a concept $i$ on bipolar scale $j$ given by personality $k$ is modeled as

$$
z_{i j k}=\sum_{p=1}^{s} \sum_{q=1}^{t} \sum_{r=1}^{u} a_{i p} b_{j q} c_{k r} g_{p q r}+e_{i j k},
$$

where $a_{i p}$ is the component loading of concept $i$ on the $p$ th concept component,

$b_{j q}$ is the component loading of scale $j$ on the $q$ th scale component,

$c_{l r}$ is the component loading of personality $k$ on the $r$ th personality component;

$g_{p q r}$ is the pqrth element of the core matrix, and indicates the importance and direction of the relationship between the pth, qth, and rth components, and

$e_{i j k}$ is the error of approximation or residual from the model.

The importance of a particular combination of components may be assessed from $g_{p q r}^{2} / S S$ (Total), where SS(Total) is the total sum of squares present in the data, and the $\Sigma_{p} \Sigma_{q} \Sigma_{r} g_{p q r}^{2}$ is equal to SS(Fit), the overall fit of the model for the specified number of components. Each $g_{p q r}^{2} / S S$ (Total) indicates the amount of explained sum of squares by that particular combination of components. For example, Table 5 shows that in the present data, $31 \%$ of SS(Total) is explained by the combination of the first components from the three modes. The $g_{p q r}$ may also be interpreted as the score for an idealized (or latent) concept $p$ on an idealized (or latent) scale $q$ by an idealized personality $r$, where an idealized entity has a nonzero loading on only one component (see Kroonenberg, 1983b, chap. 6 for further interpretations of the core matrix). 


\section{Analyzing Concept-Scale Interactions By Way of Joint Plots}

As mentioned above, the core matrix contains the information about the interactions of the components of the three modes. One of the ways to investigate individual differences in the conceptscale interactions is to examine these interactions for each subject component, or idealized subject, separately. In particular, the elements of the $r$ th "core plane," $\mathbb{G}_{r}=\left\{g_{p q r} / p=1, \ldots, s ; q=1, \ldots\right.$, t\}, represent the strength and direction of the interactions between the scale and concept components for the $r$ th idealized subject. Sometimes the interpretation of these interactions is somewhat hampered by the lack of clear labels for the components of the concepts, and occasionally (as in this example) for the components of the scales. The clear EPA structure in the scale space in most semantic differential studies is, however, often a great help in interpretation.

A way to circumvent labeling components of two modes (say, scales and concepts), sacrificing part of the parsimony of the components, is to construct a plot for each component of the remaining mode (subjects), which simultaneously displays the elements of the concepts and scales. This has the additional advantage for the interpretation that concept-scale interaction is generally thought of in terms of the actual concepts and scales rather than in terms of their components. Using the basic results of a three-mode analysis, a so-called joint plot may be constructed in which the concept-scale interactions may be visually assessed. This joint plot is a variant of Gabriel's (1971) biplot, and is perhaps best explained by way of a digression describing the biplot. The major results derived for the biplot are also valid for the joint plot.

\section{Bipplot}

The biplot is a graphic display of a matrix $\mathbb{X}$ with $\mathbb{I}$ rows and $J$ columns by means of markers $\mathbb{a}_{1}, \mathbb{a}_{2}, \ldots, \mathbb{a}_{I}$ for its rows and markers $\mathbb{b}_{1}, \mathbb{b}_{2}, \ldots$, ${ }_{j}$ for its columns. These markers are chosen in such a way that the inner product $\mathbf{a}_{i}^{\prime} \mathbf{b}_{j}$ represents $x_{i j}$, the $i j$ th element of (Gabriel, 1981, p. 147).
By assembling the markers as rows of a matrix $\mathbf{A}$, and the markers as rows of a matrix $\mathbb{R}^{2}$, this inner-product relationship implies that $\mathbb{A B}^{\prime}$ represents the matrix $\mathbf{X}$ itself. A low-dimensional representation of dimension $v(=2,3)$ suitable for plotting is called an approximate biplot of the original matrix $\mathbb{X}$, because no longer does $\mathbb{X}=\mathbb{A} \mathbb{B}^{\prime}$, but $\mathbb{X} \sim \mathbb{A}_{(v)} \mathbb{B}_{(v)}^{\prime}$, where " $\sim$ " indicates a least-squares approximation with $\mathbb{A}_{(v)}$ and $\mathbb{B}_{(v)}$ of rank $v$.

The most common forms of the biplot are: (1) $\mathbb{A}=\mathbb{U} \mathbb{A}$ and $\mathbf{B}=\mathbf{V}$, (2) $\mathbb{A}=\mathbb{U}$ and $\mathbb{B}=\mathbb{V} \mathbf{A}$, or (3) $\mathbf{A}=\mathbb{U} \mathbb{A}^{1 / 2}$ and $\mathbb{B}=\mathbb{V} \mathbb{M}^{1 / 2}$, where $\mathbb{X}=\mathbb{U} \mathbb{V}^{\prime}$ is the singular value decomposition of $\mathbb{X}$ with $\mathbb{U}$ the left eigenvector matrix of $\mathrm{X}, \mathrm{V}$ is the right eigenvector matrix, and $\mathbb{\Lambda}$ is the diagonal matrix with singular values or roots of the eigenvalues of $\mathrm{XX}^{\prime}$ and $\mathbf{X}^{\prime} \mathbf{X}$. From the three forms above, it can be seen that the elements of $\mathbb{A}$ act as scaling constants for the eigenvectors in $\mathbb{U}$ and/or $\mathbb{V}$.

By simultaneously displaying column and row markers (i.e., the elements of two modes) in one plot, visual inferences can be made about their relationships, that is, about the structure of the matrix $\mathbf{X}$. The basis for this assertion is that an inner-product of two vectors may be assessed visually by considering it as the product of the length of one of the vectors times the length of the other vector's projection onto it. The relationship of two vectors with respect to a third can thus be assessed simply by comparing their projections onto that third vector. It can also easily be seen which rows or columns are proportional to what other rows and columns, which rows and columns are at right angles, that is, have an inner-product of zero, and thus a zero value in $X$, and so forth. In semantic differential studies it is generally convenient to represent the elements of one mode (say, scales) by vectors through the origin, and those of the other mode (say, concepts) by points, if only to distinguish between the two. Concepts with large projections onto the positive side of a vector (scale) have high scores on that scale, concepts with small projections have scores near the neutral point of the scale (given that the scales are centered at the neutral point), and concepts with large projections on the negative side of a scale have low scores on 
that scale. This and other uses of the biplot have been extensively discussed and illustrated in Gabriel (1981).

\section{Toint Plots}

In three-mode analysis for each subject component, or idealized subject, there is a slice or plane of the core matrix, $G_{r}$, which indicates the relationship between the scale and concept components. To investigate the relationships between the actual concepts and scales, this core plane may be combined with the common concept and scale spaces $=\left(a_{i p}\right)$ and $=\left(b_{j q}\right)$, such that $\mathbb{R}_{r}=\mathrm{G}_{r} \mathrm{~B}^{\prime}$ (which has the order of $I$ concepts by $J$ scales), and make some form of biplot of 1 , for each idealized subject $r$. For the form of biplot, which is called a joint plot, the core plane $G$, is exactly decomposed by way of the singular value decomposition as $\mathbb{G}_{r}=\mathbb{U}_{r} \mathbb{A}_{r} V_{r}$ ', and the 'marker matrices" are constructed as

$$
\begin{aligned}
& \tilde{\mathbb{A}}_{r}=\left(\frac{I}{J}\right)^{1 / 4} \mathrm{AU}_{r} \mathrm{~A}_{r}^{1 / 2}, \\
& \tilde{\mathbb{B}}_{r}=\left(\frac{J}{I}\right)^{1 / 4} \mathbb{R}_{r} \mathrm{~A}_{r}^{1 / 2},
\end{aligned}
$$

thus using the third variant of the biplot mentioned above with extra scaling constants to make the lengths of the marker vectors more comparable. The inner products,

$d_{i j}^{(r)}=\tilde{q}_{i}^{(r)} \tilde{H}_{j}^{(r)}$,

are thus visually inspected to assess the conceptscale interactions, and can also be compared numerically. For each component $r(r=1, \ldots, u)$, a joint plot can be made using every time the common $\mathbb{A}$ and $\mathbb{R}$, and the idiosyncratic core plane $\mathbb{G}_{r}$. In the TUCKALS3 program joint plots can be made for any combination of two modes given a core plane associated with a component of the remaining mode, but it will seldom be necessary to use all possible combinations. As biplots, joint plots are only really useful when the dimensionality of $A$ and $\mathbb{B}$ is rather low, say, 2 or 3 . When $\mathbb{A}$ and $\mathbb{B}$ have a different number of components, say $s$ and $t$ with $s<t$, the joint plot can only be made in $s$ dimensions. Note that this does not automatically mean that the $t-s$ last components of are discarded. The particular structure of the core plane $\mathbb{G}_{r}$ will determine how an $s$-dimensional subspace is selected from the $t$-dimensional space of

\section{Assessing ihe Quality of

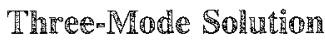

When the parameters in the model of Equation 2 are solved by way of alternating (or conditional) least squares procedures (for details see Kroonenberg \& de Leeuw, 1980; Kroonenberg, 1983b), it is possible to partition the loss function:

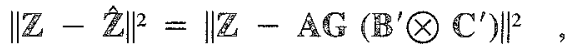

in such a way that

$\underset{i j k}{\sum \sum_{j} \sum_{i j k}}=\frac{\sum_{i j k} \sum_{j} \hat{z}_{i j k}^{2}}{\sum_{i}} \sum_{j k} \sum_{i j k} e^{2}$

or

$S S($ Total $)=S S($ Fit $)+S S($ Res $)$.

Furthermore, it is possible to show that for each element $f$ of a mode (say, for the concept DOCTOR, the scale active-passive, or the subject $k$ ), it is true that

$S S\left(\operatorname{Total}_{f}\right)=S S\left(\mathrm{Fit}_{f}\right)+S S\left(\operatorname{Res}_{f}\right)$.

In other words, it is possible to determine how well the data of an element of a mode are represented by the model, and to compare how well elements of a mode have been fitted relative to each other. This property is extremely helpfull in searching for outliers, overly influential points, and so forth. Furthermore, using quantities like SS(Fit $) / S S\left(T o t a l_{f}\right)$, the adequacy of the three-mode solution can be compared to the solution obtained by analyzing the data of that element (say, subject) separately.

The sums-of-squares partitioning is also helpful in choosing the number of components in each of the three modes. Given that three-mode analysis is intended primarily for data-analytic or exploratory purposes, the choice is not as critical as in (confirmatory) factor analysis, and the decision depends largely on the detail and compactness with which the data are to be described. Notwithstanding, some guidance as to the adequacy of the description is necessary. The primary information is, as in stan- 
dard (two-mode) principal components analysis, the amount of variation (i.e., sums of squares) contributed by each of the components in a mode. Two problems make the decisions rather complicated. First, due to the estimation of the parameters by way of minimizing loss functions such as Equation 6 , only the user-specified numbers of components for each mode are available from a single analysis, whereas in standard principal components analysis usually the contribution of all components is available. Aids for deciding the adequate number of components such as Cattell's scree test can, therefore, not be used. Secondly, the previous problem is aggravated because solutions with different numbers of components are not nested, that is, allowing for an extra component in a mode does not only give estimates for that new component, but also affects the parameters in the other components as well, unlike standard principal components analysis. In fact, changing the number of components in any mode may affect the solution in all three modes. When the data are well-structured, this lack of nesting is not always noticeable, or problematic, but it makes developing clear guidelines for choosing an adequate number of components very difficult. Often, therefore, it is necessary to rely on knowledge of the subject matter, comparison between various solutions, and some "artistry" in choosing an adequate solution.

In particularly difficult cases it is often helpful to explicitly use the three-mode nature of the solution. As mentioned above, each $g_{p q r}^{2}$ of the core matrix indicates the amount of fitted sums of squares by the $p, q, r$-combination of components. Furthermore,

$$
S S\left(\mathrm{Fit}_{p}\right)=\sum_{q=1}^{t} \sum_{r=1}^{u} g_{p q r}^{2}
$$

is equal to the amount of fitted variation by the $p$ th component of the frrst mode, and similarly for summations over other pairs of indices. Adding an extra component, say $p+1$, gives an extra set of $g_{p+1, q, r}(q=1, \ldots, s ; r=1, \ldots, u)$ elements in the core matrix, but the increase in fir might be due to only one particular combination of components, say $p+\mathbb{1}, q^{\prime}, r^{\prime}$. In such cases the size of $g_{p+1, q^{\prime}, r^{\prime}}^{2}$ relative to the other $g_{p q r}^{2}$ can be used to assess whether the extra component is worthwhile.

\section{Preprocessing of Senmantic Differential Data}

As pointed out by Kroonenberg (1983b, p. 128ff), and Harshman and Lundy (1984), a central question for any three-mode analysis is the treatment of the means before the analysis proper. As it is assumed in semantic differential research that the center of the scale is the neutral point, and that a concept at that center on all scales is a "meaningless" concept (cf. Osgood \& Luria, 1954/1969, p. 507 ), it seems most proper to subtract the scale midpoint 4 from all values-a procedure also followed by, for example, Levin (1965) and Snyder and Wiggins (1970). An alternative would be to compute per administration standard scores for each concept or scale, as was probably done during the factor analyses of Osgood and Luria. The disadvantage of the latter approach is that shifts in overall level of scoring between subjects are eliminated from the analysis.

\section{Theree-Mode Analysis of a Triple Personality}

After investigating solutions with varying numbers of components for the three modes, it was decided to report the details of the $2 \times 3 \times 2$ solution, that is, the solution with 2 scale components, 3 concept components, and 2 personality components. The results for each of the component spaces are reported first, followed by those of the interactions of these components as found in the core matrix.

\section{Scale Space}

In contrast to most analyses of semantic differential data only two scale components, $\$ 1$ and $\$ 2$, were deemed sufficient, explaining $59 \%$ and $11 \%$ of the SS(Total), respectively. With these two components most similarities and differences between the personalities can be described; the third scale component, contrasting fast versus large, clean, and valuable, explains only another $3 \%$ of the total variation. Table 1 shows the two-dimensional scale 
Table 1

Componemt Loadings of Scales for $2 \times 3 \times 2$ Solution

\begin{tabular}{lccc}
\hline Scale & Dimension & S1 & S2 \\
\hline Valuable-worthless & $\mathrm{E}$ & .42 & .01 \\
Clean-dirty & $\mathrm{E}$ & .39 & .12 \\
Tasty-distasteful & $\mathrm{E}$ & .38 & -.23 \\
Fast-slow & $\mathrm{A}$ & .05 & .25 \\
Active-passive & $\mathrm{A}$ & .31 & .45 \\
Hot-cold & $\mathrm{A}$ & .25 & -.18 \\
Large-smal1 & $\mathrm{P}$ & .34 & .08 \\
Strong-weak & $\mathrm{P}$ & .33 & -.26 \\
Deep-shallow & $\mathrm{P}$ & .30 & .42 \\
Relaxed-tense & $\mathrm{E}, \mathrm{A}$ & .21 & -.62 \\
\% Explained & & & \\
Variation & & 59 & 11 \\
\hline
\end{tabular}

Note: $E=$ Evaluation; $P=$ Potency; $A=$ Activity

space in which the absence of an EPA structure is conspicuous.

It is possible that the particular preprocessing procedure was responsible for the lack of EPA structure. However, using the same centering procedure, the analyses by Levin (1965) and Snyder and Wiggins (1970) showed a clear EPA structure after rotation; furthermore, varimax rotation for the two-component solution will not succeed in arriving at the desired structure. An attempt was also made to apply varimax to the three-component solution, but this also failed to produce EPA dimensions. To investigate the effect of preprocessing itself, two different kinds of preprocessing were applied to the data: (1) removing scale and concept means per administration, and (2) removing only scale means per administration. However, neither case produced an EPA structure, leading to the conclusion that the EPA dimensions as given in Osgood and Luria $(1954 / 1969$, p. 506) for the specific semantic differential scales used in their study, are not used by Eve White, Eve Black, and Jane in the same way as other subjects do in psychotherapy studies.

\section{Concept Space}

In comparison with Osgood and Luria's (1954/ 1969) indirect way of deriving the concept space (see above), the configuration of concepts emerges naturally in three-mode analysis, and its dimensionality can be assessed independently of the dimensionality of the scale space. Three dimensions $(\mathrm{C} 1, \mathrm{C} 2$, and $\mathrm{C} 3$ ), explaining $38 \%, 21 \%$, and $10 \%$ of the SS(Total), respectively, were necessary to give a reasonable representation of the concept space (Table 2).

\section{Persomalinky Space}

The overall similarities and differences between the administrations are succinctly described by the personality space, that is, by the loadings on the two components ( $\mathbb{P} 1$ and $\mathrm{P} 2$ ), which explain $45 \%$ and $23 \%$ of the SS(Total), respectively. In Table 3 the principal axes were orthonomally rotated over an angle of $10^{\circ}$ to let the rotated axes coincide as much as possible with the personalities. The table shows that Eve White and Jane determine the first axis (P1), are highly similar in both testings and similar to each other, and that Eve Black $\mathbb{I}$ and II have the second axis (P2) to themselves. Apart from error or not-fitted sum of squares, Jane's data are not very different from those of Eve White, and have a very different pattern from those of Eve Black, indicating that Jane (the "terminal personality") bears very little resemblance to Eve Black, and seems to have evolved almost entirely from 
Table 2

Component Loadings of Concepts for $2 \times 3 \times 2$ Solution

\begin{tabular}{lccc}
\hline Concept & C1 & C2 & C3 \\
\hline my DOCTOR & .23 & .53 & -.01 \\
PEACE of mind & .19 & .45 & .20 \\
my FATHER & .26 & .31 & -.21 \\
self-CONTROL & .28 & .19 & .01 \\
my MOTHER & .24 & .17 & -.32 \\
my CHILD & .31 & -.06 & -.13 \\
LOVE & .39 & -.14 & .05 \\
my JOB & .30 & -.16 & -.08 \\
my SPOUSE & .25 & -.17 & .08 \\
mental SICKNESS & .23 & -.23 & -.38 \\
SEX & .26 & -.25 & .05 \\
CONFUSION & .11 & -.23 & -.50 \\
ME & -.08 & .30 & -.24 \\
HATRED & -.27 & .11 & -.43 \\
FRAUD & -.30 & .08 & -.38 \\
\% Explained & & & \\
Variation & 38 & 21 & 10 \\
\hline
\end{tabular}

Eve White, as far as this can be judged on the basis of the semantic differential data. What difference there is between Eve White and Jane might be judged from the third personality component, but it explains not even $1 \%$ of the SS(Total), and is not further discussed in this paper.

The validity of the results could be questioned on the grounds that, even though the three-mode results indicate the commonalities of the person- alities, it might give a very distorted view of each of the personalities taken separately. In other words, it could be that the technique allows an assessment of what the personalities have in common, but this might not be very much. If this were true, substantial discrepancies in fit should exist between the principal components solutions for each personality separately (here also computed using the TUCKALS3 program), and the fit of each person-

Table 3

Component Loadings of Personalities

for $2 \times 3 \times 2$ Solution

\begin{tabular}{|c|c|c|}
\hline Personality & $\mathrm{P} 1$ & $\mathrm{P} 2$ \\
\hline Eve Black I & .00 & -.63 \\
\hline Eve Black II & .00 & -.77 \\
\hline Eve White I & .49 & -.02 \\
\hline Eve White II & .46 & -.08 \\
\hline Jane I & .51 & .02 \\
\hline Jane $\quad$ II & .54 & .06 \\
\hline \multicolumn{3}{|l|}{$\%$ Explained } \\
\hline Variation & 45 & 23 \\
\hline
\end{tabular}

Note: $I=f i r s t$ testing, II=second testing

Downloaded from the Digital Conservancy at the University of Minnesota, http://purl.umn.edu/93227. 
Table 4

Comparison of Separate Fit with Fit from Three-mode Analys is

\begin{tabular}{lrccc}
\hline \multirow{2}{*}{ Personality } & $\begin{array}{c}\text { Three-Mode } \\
\text { Fit }\end{array}$ & $\begin{array}{c}\text { Separate } \\
\text { Fit }\end{array}$ & $\begin{array}{c}\text { Difference } \\
\text { in Fit }\end{array}$ \\
\hline \multirow{2}{*}{ Eve White } & I & .65 & .72 & .08 \\
\multirow{2}{*}{ Eve Black } & II & .66 & .80 & .14 \\
\multirow{2}{*}{ Jane } & II & .58 & .79 & .21 \\
& I & .81 & .92 & .11 \\
& II & .70 & .78 & .08 \\
\hline
\end{tabular}

ality in the three-mode principal components solution. Such information is contained in Table 4 , in which the fit is expressed as proportions explained sums of squares. As Table 4 shows, differences exist but they stay within reasonable bounds, except perhaps for Eve Black II. It seems fair to say that the overall solution succeeds reasonably well in simultaneously describing all six administrations of the semantic differential.

\section{Concept-Scale Triterartion}

As mentioned above, the information about the interactions between concepts, scales, and personalities is contained in the core matrix. In particular, the elements of the $r$ th "core plane" $G_{r}$ represent the strength and direction of the interactions between the scale and concept components for the rth personality component. As, after rotation, the first personality component is exclusively determined by Eve White and Jane, $\mathbb{G}_{1}$ (Table 5A) describes how they relate the scale and concept components, $\mathbb{G}_{2}$ (Table 5B) does the same for Eve Black. From Tables $5 \mathrm{~A}$ and $5 \mathrm{~B}$ it is clear that Eve White/Jane and Eve Black primarily use the evalluation scale component ( $\$ 1 ; 99 \%$ and $94 \%$ of their SS(Fit), respectively), but that they differ with respect to the concepts corresponding with the evaluation axis of the scale space. For Eve White/Jane this is primarily the first concept component contrasting HATRED and FRAUD with most other concepts; for Eve Black this is predominantly the

Table 5

Core Matrix

\begin{tabular}{|c|c|c|c|c|}
\hline \multirow{2}{*}{$\begin{array}{l}\text { Concept } \\
\text { Component }\end{array}$} & \multicolumn{2}{|c|}{$\begin{array}{c}\text { A } \\
\text { Personality Component } 1 \\
\text { (Eve White \& Jane) }\end{array}$} & \multicolumn{2}{|c|}{$\begin{array}{c}\text { B } \\
\text { Personality Component } 2 \\
\text { (Eve Black) }\end{array}$} \\
\hline & $\mathrm{S1}$ & S2 & $\widehat{\mathrm{S}} 1$ & S2 \\
\hline$\overline{\mathrm{Cl}}$ & 16.8 & 0.6 & 7.3 & -1.8 \\
\hline $\mathrm{C} 2$ & 5.5 & $=0.7$ & 12.7 & -0.7 \\
\hline $\mathrm{C} 3$ & -0.0 & -1.5 & -0.5 & 3.7 \\
\hline $\begin{array}{l}\% \mathrm{Ex} \\
\text { Comb }\end{array}$ & $\begin{array}{l}\text { hed Variation } \\
\text { Lon of Compone }\end{array}$ & & & \\
\hline $\mathrm{C} 1$ & 31 & 0 & 6 & 0 \\
\hline $\mathrm{C} 2$ & 3 & 0 & 18 & 0 \\
\hline $\mathrm{C3}$ & 0 & 8 & 0 & 1 \\
\hline
\end{tabular}

Note: S1. S2 indicate first. second scale component respectively 
second component contrasting DOCTOR, PEACE, ME, and FATHER with mental illness and day-today concepts. Clearly the second scale component is not very important but more so for Eve Black than Eve White/Jane (6\% and $1 \%$ of their SS(Fit), respectively).

Eve Black. Eve Black's (Personality Component 2) scale and concept relationships are given by a joint plot in Figure 1. Summarizing the relationships (using the interpretational rules given above), it could be said that all concepts related to day-to-day life (IOB, SPOUSE, CHILD, SEX, LOVE) are evaluated negatively and are considered neutral with respect to scales such as active, deep, and relaxed. Those concepts related to Eve Black's mental make-up (CONFUSION and SICKNESS) are also evaluated negatively, but somewhat active and deep, and rather tense as well. Eve Black regards with favor her DOCTOR, ME, PEACE, HATRED, and FRAUD, and has a moderately fa- vorable opinion of her parents, as well as a moderately active and deep, and a rather tense judgment of them.

Eve White and Jane. Unlike Eve Black, Eve White and Jane (Personality Component 1) seem to be reasonably "mormal" (see Figure 2). All concepts related to day-to-day life and therapy are positively evaluated, whereas HATRED and FRAUD are not. $\mathrm{ME}$ is seen as a neither good nor bad concept and somewhat fast, weak, and distasteful, as well as rather tense, active, and deep. Furthermore, note that CONFUSION and SICKNESS are meutrally evaluated, and are very tense, active, weak, distasteful, and cold.

The sums-of-squares interpretation allows the evaluation of statements like "Jane is becoming less diversified semantically (more 'simple-minded') rather than the reverse" (Osgood \& Luria, 1954/ 1969 , p. 516), with " . . all of her judgments tending to fall along a single factor of good-strong

Tigigure 1

Joint Plot of Eve Black's Concept-Scale Space

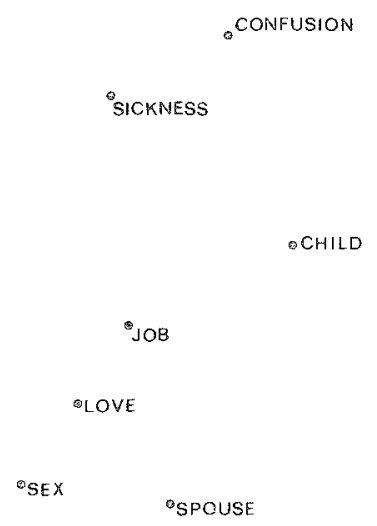

Q MOTHER

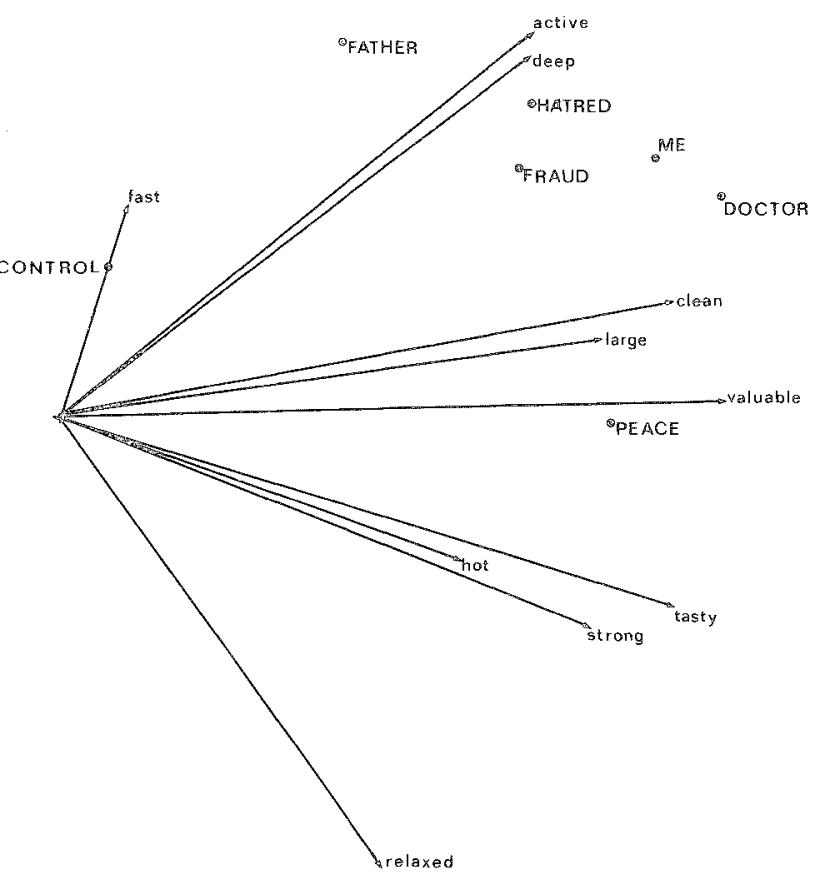

Downloaded from the Digital Conservancy at the University of Minnesota, http://purl.umn.edu/93227.

May be reproduced with no cost by students and faculty for academic use. Non-academic reproduction requires payment of royalties through the Copyright Clearance Center, http://www.copyright.com/ 


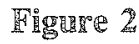

Joint Plot of Eve White's and Jane's Concept-Scale Space

- CONFUSION

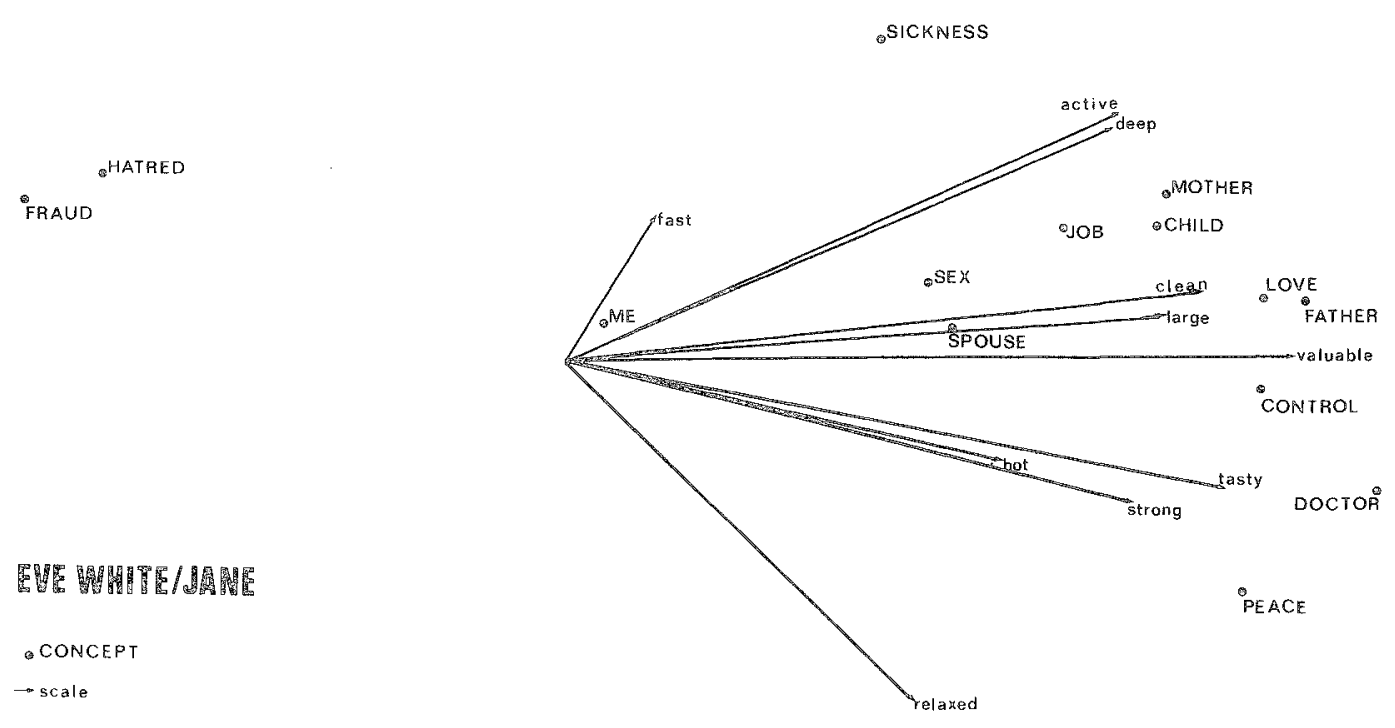

vs. bad-weak" (p. 514). This conclusion is only very weakly supported by the present analysis, as can be demonstrated by examining the relative importance of the various components for each of the administrations as expressed by the fitted sums of squares. In Table 6 this fitted sum of squares or fit is expressed as the proportion explained sum of squares of the total sum of squares of each personality. (The entries are derived from Table 9.7 in Kroonenberg, 1983b, p. 237.) If "simple-mindedness" means that one of the scale components increases at the cost of the other, then indeed it can be observed from Table 6 that Jane's fit for the first component increases from .52 to .70 , and the fit for the other component decreases from .17 to .11 . Whether this change warrants the fairly strong statement of Osgood and Luria is rather doubtul. The statement that there is an "increasing simplification in structure characteristic of all three personalities" (p.517) cannot be supported in the same manner (see Table 6).

Table 6

Fit of Scale Components for Each Personality

\begin{tabular}{lccc}
\hline & & Fit of Component \\
\cline { 2 - 3 } Personality & S1 & S2 \\
\hline \multirow{2}{*}{ Eve White I } & .48 & .17 \\
\multicolumn{2}{l}{ Eve Black II } & .49 & .18 \\
\multirow{2}{*}{ Jane } & II & .56 & .02 \\
& II & .77 & .04 \\
& II & .52 & .17 \\
\hline
\end{tabular}

Downloaded from the Digital Conservancy at the University of Minnesota, http://purl.umn.edu/93227. May be reproduced with no cost by students and faculty for academic use. Non-academic reproduction requires payment of royalties through the Copyright Clearance Center, http://www.copyright.com/ 


\section{Discanssion}

This paper attempted to show how three-mode principal components analysis can be fruitfully used to provide a description of the individual differences in scale and concept usage in semantic differential data. In particular, it was shown that such individual differences can be handled by three-mode principal components analysis far more easily than was customary. Especially the simultaneous plotting of concepts and scales for each idealized subject (or here, personality) marks an advance over the usual analyses, whether three-mode or not. It allows a more detailed and comprehensive interpretation, especially in those cases, as here, in which an EPA structure is absent, or generally when it is difficult to label components. Furthermore, it becomes possible to describe individual differences by aspects common or invariant over personalities, and by interactions particular to each of them, yielding, on the whole, a fairly parsimonious description. Finally, the sums-of-squares interpretation of the core matrix and the possibility of assessing the relative fit of virtually all parts of the model gives considerable control over the outcome of the analysis.

\section{Rei̊erences}

Gabriel, K. R. (1971). The biplot graphical display of matrices with application to principal component analysis. Biometrika, 58, 452-467.

Gabriel, K. R. (1981). Biplot display of multivariate matrices for inspection of data and diagnosis. In $\mathrm{V}$. Bamett (Ed.), Interpreting multivariate data (pp. 147 164). Chichester, England: Wiley.

Harshman, R. A., \& Lundy, M. E. (1984). Data preprocessing and the extended PARAFAC model. In H.G. Law, C. W. Snyder Ir., J. A. Hattie, \& R. P. McDonald (Eds.), Research methods for multi-mode data analysis (pp. 216-284). New York: Preager.

Heise, D. R. (1969). Some methodological issues in semantic differential research. Psychological Bulletin, $72,406-422$.

Kroonenberg, P. M. (1981). User's guide to TUCKALS3. A program for three-mode principal component analysis (WEP Reeks, WR 81-6-RP, Section W.E.P.). Leiden, The Netherlands: University of Leiden, Department of Education.
Kroonenberg, P. M. (1983a). Annotated bibliography of three-mode factor analysis. British Journal of Mathematical and Statistical Psychology, 36, 81-113.

Kroonenberg, P. M. (1983b). Three-mode principal component analysis: Theory and applications. Leiden, The Netherlands: DSWO Press.

Kroonenberg, P.M., \& de Leeuw, J. (1980). Principal component analysis of three-mode data by means of alternating least squares algorithms. Psychometrika, $45,69-97$.

Levin, J. (1965). Three-mode factor analysis. Psychological Bulletin, 64, 442-452.

Osgood, C. E., \& Luria, Z. (1969). A blind analysis of a case of multiple personality. In J. G. Snider \& C. E. Osgood (Eds.), Semantic differential technique: $A$ source book (pp. 505-517). Chicago IL: Aldine. (Reprinted from Journal of Abnormal and Social Psychology, 1954, 49, 579-591)

Osgood, C. E., Suci, G. J., \& Tannenbaum, P. (1957) The measurement of meaning. Urbana IL: University of Illinois Press.

Snider, J. G., \& Osgood, C. E. (Eds.). (1969). Sernantic differential technique: A source book. Chicago IL: Aldine.

Snyder, F. W., \& Wiggins, N. (1970). Affective meaning systems: A multivariate approach. Multivariate Behavioral Research, 5, 453-468.

Thigpen, C. H., \& Cleckley, H. (1954). A case of multiple personality. Journal of Abnormal and Social Psychology, 49, 135-151.

Tucker, L. R. (1963). Implications of factor analysis of three-way marrices for the measurement of change. In C. W. Harris (Ed.), Problems in measuring change (pp. 122-137). Madison: University of Wisconsin Press. Tucker, I. R. (1966). Some mathematical notes on threemode factor analysis. Psychometrika, 31, 279-311.

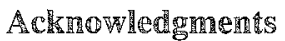

An earlier version of this article was published as part of the author's doctoral thesis by the DSWO Press, Middelstegracht 4, Leiden. The author thanks Jan de Leeuw for suggesting the analysis, and Wim van der Kloot for commenting on the manuscript.

\section{Author'\$ Address}

Send requests for reprints or further information to Pieter M. Kroonenberg, Department of Education, University of Leiden, P.O. Box 9507, 2300 RA Leiden, The Netherlands. 